Proceedings of the 2012 Winter Simulation Conference

C. Laroque, J. Himmelspach, R. Pasupathy, O. Rose, and A.M. Uhrmacher, eds

\title{
A SIMULATION STUDY OF PATIENT FLOW FOR DAY OF SURGERY ADMISSION
}

\author{
Michael E. Kuhl \\ Rochester Institute of Technology \\ Industrial and Systems Engineering \\ 81 Lomb Memorial Drive \\ Rochester, NY 14623, USA
}

\begin{abstract}
In this paper the patient flow and perioperative processes involved in day of surgery admissions are considered for a hospital that is undergoing a staged redesign of its operating room. In particular, the day of surgery admission area where patients are prepared for surgery is being relocated and some additional functions for the new unit are being considered. The goal of the simulation study is to map the patient flows and functions of the current area into the newly designed space, to measure potential changes in productivity, and to determine opportunities for future improvements.
\end{abstract}

\section{INTRODUCTION}

Perioperative care in a hospital encompasses all of the medical and related processes involved in surgery form the time the patient arrives, through the surgical procedure, and recovery, until either the patient is discharged or admitted to a hospital room. One integral aspect of perioperative care is day of surgery admission. In this paper, day of surgery admission (DOSA) will refer to the process of admitting patients on the day of their scheduled surgery and preparing the patient for surgery. This process concludes when the patient is taken into the operating room. The DOSA process is important to the overall functioning and productivity of the operating room and is a critical step in ensuring high quality patient care and safety.

In this case study, the focus will be on the patient flow and productivity aspects of the day of surgery admission process. In particular, the hospital that is serving as the basis for this study is undergoing a staged redesign of their perioperative space including construction and reconfiguration of operating rooms and related functional areas. The hospital is a 528-bed facility located in western New York state. The DOSA unit serves the admission of patients for general surgery which take place in 15 of the hospital's operating rooms (other ORs are utilized for ambulatory surgery and other outpatient procedures) and is limited to patients entering the hospital on the day of surgery (emergency and in-patient surgical patients are not prepared for surgery in DOSA). As part of the first phase of the redesign, the DOSA unit is planned to be relocated and reconfigured. The goal of this simulation study is to evaluate how the new configuration will impact patient flow and work flow and to determine opportunities for future improvement.

In recent years, there has been a number of simulation studies related to various aspects of operating room and perioperative processes. In particular, Denton et al. (2010) investigate the optimal allocation of surgery blocks for operating rooms utilizing block scheduling policies, and Marjamaa et al. (2009) utilize simulation to study workflow in the operating room. In addition, Ballard and Kuhl (2006) utilize simulation to conduct a capacity analysis of surgical suite of operating rooms, and Segev et al. (2012) conduct a simulation study of patient transportation and the impacts productivity of perioperative processes. Although these papers address various aspects of the perioperative process, they do not directly address day of surgery admission which we undertake in this paper. 
The remainder of the paper is organized as follows. Section 2 provides an overview of the case study including the current and planned DOSA unit configurations. Section 3 outlines the simulation methodology. Section 4 presents experimental results, and conclusions and future work are discussed in Section 5.

\section{DOSA CASE STUDY}

This case study focuses on the Day of Surgery Admission Unit which will be relocated to a newly reconstructed area of the surgical floor of the hospital. The purpose of this project is aid in establishing an efficient, patient-centered day of surgery admission process in the new DOSA location. The DOSA unit currently operates in an area of multi-patient rooms containing a total of 15 beds and the new area is planned to include 18 three-walled DOSA beds within an enclosed suite.

The objectives of this analysis are to model the current patient and process flow in the current DOSA area and identify areas of potential improvement in efficiency and patient-centered care; model the future patient and process flow in the newly constructed DOSA area; and identify, model, and quantify alternatives for improvement of patient and process flows and their impact on the key performance indicators including patient waiting times, utilization of nurses and DOSA staff, patient throughput, and DOSA workday completion time.

To model the patient and process flows, discrete event simulation will be used. The simulation model will be designed to represent the dynamic behavior of the DOSA unit including the arrival and flow of patients through the DOSA process and work flow of DOSA staff. In addition to DOSA, the model will encompass some aspects of related areas that directly interact with and impact the DOSA processes and patient flow. Statistical models will be used to represent the variability of process times in the system. The simulation model will produce statistical output relative to the key performance indicators; and appropriate design of experiment and statistical analysis methods will be used to compare alternative systems.

\subsection{DOSA Patient Flow}

The patient flow through the DOSA unit that we will consider begins when the patient arrives to the hospital and concludes when the patient is sent into the operating room. A flowchart illustrating this patient flow in the current system is presented in Figure 1.

When a patient enters the hospital for surgery, they are greeted near the main entrance at what the hospital refers to as the Patient Access Center. Patients are asked to arrive two hours prior to their scheduled surgery time. There are two types of patients that arrive - standard admits and direct admits. Standard admit patients go through a pre-admission process typically 1-2 weeks prior to their date of surgery which includes a history and physical and other pre-admission testing ordered by their physician as well as financial payment and insurance coverage arrangements. These standard admit patients are sent immediately to the DOSA unit. Direct admit patients meet with an admitter at the Patient Access Center to complete appropriate admission and financial form and are then sent to the DOSA unit.

Upon reaching the DOSA unit, patients check-in with the receptionist and wait until a nurse or technician (tech) brings them to a room to begin their preop process. The preop process consists of, first, the patient changing into a patient gown. Then, the nurse begins an assessment of the patient including a discussion of the surgery to be done, taking vital signs, administering an IV, interviewing the patient about their medical history, etc. Then the nurse (if available, with the help of a tech) implements the orders provided by the physician which may include various tests (EKG, lab work, x-rays, etc.) and administers any required medication. Direct admit patients may also require a history and physical exam. Upon the completion of the preop process, in the current system, the patient waits in DOSA until the patient is called for by OR Holding and a transporter is sent to move the patient.

In OR Holding, a nurse waits with the patient. When appropriate the anesthesiologist will meet with the patient to discuss the surgery and then the surgeon will meet with the surgery and the risks as well as obtain consent from the patient. Once this is complete and the operating room is ready, the patient is taken into the OR. 


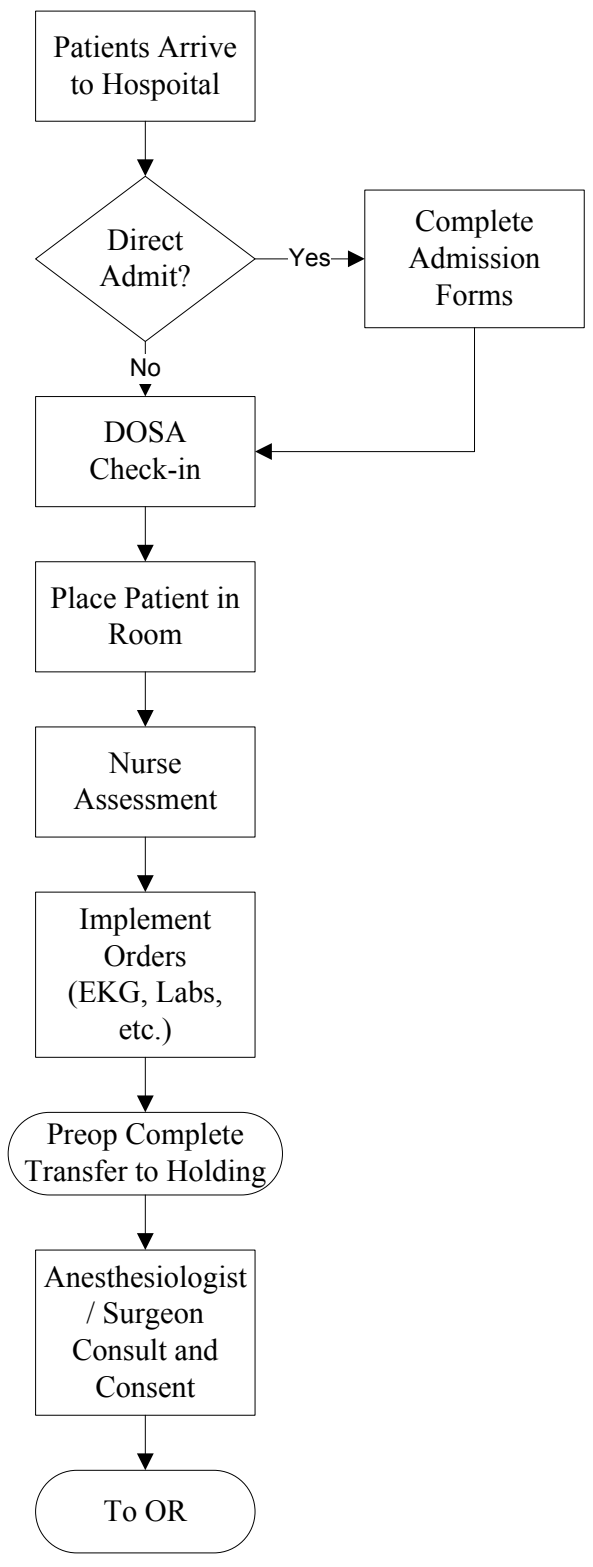

Figure 1: Patient flow through day of surgery admission process

For the DOSA process, there are several events that could potentially cause bottlenecks for a particular case as well as a domino effect that can occur for the process on a particular day. Since the process essentially starts over each day, the is minimal (if any) impact of one surgical day to the next. The potential delays or bottlenecks could be caused by the following (among others):

- Late arrival of patients for their scheduled admission time;

- Exam or lab results that require additional tests;

- Delay in receiving lab results;

- Longer than expected surgery times that could cause a patients to wait in DOSA or holding;

- Emergency surgery cases that preempt elective surgeries;

- Shortage of DOSA or OR personnel due to unexpected absences; and 
- Unavailability of beds in post-operative recovery and/or other hospital units.

Therefore, the DOSA configuration including the number of beds within the unit as well as their use can impact the performance of the unit.

\subsection{Current and Future DOSA Configurations}

The current configuration of DOSA provides room for 15 patients including 12 stretchers (four rooms containing 2 stretchers each, and one room containing 4 stretchers) and 3 individual rooms with chairs. Patients are placed in the rooms with chairs only if capable and get on a stretcher just prior to being transported to OR Holding. The multi-patient rooms containing stretchers are divided by curtains (which makes complete privacy difficult) and are restricted by gender based on the first patient placed in the room.

The future DOSA configuration is planned to have room for up to 18 patients including up to 16 individual rooms with stretchers and 2 individual rooms with chairs. This configuration will minimize privacy concerns and eliminate blocking due to gender specific room allocations. In addition, there is a desire to eliminate or reduce the OR Holding area by having the interviews by the anesthesiologist and surgeon be conducted in the DOSA room, so the patient would remain in DOSA until being transferred directly into the OR.

The OR currently operates with a scheduled case load for elective surgeries of 45-65 per day. First cases are typically scheduled at 7:30 a.m. Patients begin arriving at 5:30 a.m. and DOSA begins processing patients at 6:00 a.m. Direct admit patients are typically not scheduled to be first cases. The goal is to have all of the patients leave DOSA by 6:00 p.m. Throughout the day, ten nurses and one tech are scheduled in staggered 8 or 10 hours shifts starting at 5:30 a.m. and continuing until 6:00 p.m.

\section{MODELING METHOLOGY}

To analyze and compare the current and future system configurations, a discrete event simulation model was constructed for each system. The ARENA simulation software was selected for the analysis. The models are constructed to depict a representative case-load day in the DOSA unit. Although there are many functions that the nurses and techs perform, the model includes only those duties that are directly involved with preparing a patient for surgery. For example, nurses have the duty of calling patients that will be coming in for surgery the next day, however, this is typically done during slower times of the day when a nurse may be available or toward the end of the day when all of current day patients have been processed.

To determine the case load and the arrival processes, one year of case data was analyzed consisting of approximately 11,000 cases that were processed through the DOSA unit. (Note that this is only a portion of the total surgical cases performed at the hospital, however, these other cases are beyond the scope of this study.) The number of cases per day was determined to follow a positively skewed beta distribution with an average of 48 cases per day and a maximum of 65 cases. Of these, approximately 20 percent (up to 15 corresponding to the number of OR rooms) were determined to be first cases. For each patient, the data showed the arrival time prior to the scheduled surgery followed a normal distribution with a mean of 126 minutes and a standard deviation of 40 minutes. Of the non-first case patients, approximately $27 \%$ were direct admit patients.

Upon arrival, the patients were assigned characteristics such as gender (50\% male, 50\% female) and characteristics describing the specific physician orders the patient required including one or more of the following: EKG, x-ray, lab work, urine pregnancy test (female only), history and physical (direct admits only), etc. The patients were then processed though the DOSA unit on a first-come, first-served basis.

The model for the future system configuration has been set up to allow for use of OR Holding or to have these process be performed in the DOSA unit. 
Performance measures are collected on the waiting time of the patient, the door to nurse time in DOSA, the door to DOSA complete time, the door to OR time, the completion time of the last patient in DOSA, the completion time of the last patient in OR Holding, and the utilization of the nurses and techs.

In the model, the nurses and techs were modeled as a combination of resources and transporters. By modeling the total number of nurses collectively as resources, one can track whether or not a nurse was available. By modeling individual nurses as transporters, the movement of nurses between locations could be modeled as well as ensuring that a particular nurse once assigned to a patient would continue to serve that patient until the patient's preop processing was complete.

Verification and validation of the model was done utilizing structured walkthroughs of the model and data with DOSA nurses, nurse mangers, and operations managers. Validation of case loads was done by writing out case load scheduled produced by the simulation models and comparing them to actual case load schedules. Through this process, the simulation model was deemed to be representative of the current and planned system configurations.

\section{EXPERIMENTAL RESULTS}

In this section, we present a set of experiments to determine the performance of future DOSA configuration where individual patient rooms are utilized for both the DOSA process as well as the current functions that are performed in the holding area. A range of 15 to 18 patient rooms are considered and compared under surgical case loads varying from 50 to 80 cases per day. For each system configuration, 100 independent surgical days are simulated. Given the many other factors that can cause bottlenecks or delay within the system, the primary performance measure used to compare the configurations with different bed capacities is the Door to DOSA Complete time which is the time the patient spends in the system from the time they arrive at the hospital until the time that the DOSA preop processes are complete.

A graph comparing Door to DOSA Complete time under the various configurations and case loads appears in Figure 2. For the 15-bed case, although the current system has 15 beds, given the additional holding functions that would now be performed in the DOSA unit, the DOSA beds are utilized for longer periods of time causing arriving patients to wait and the Door to DOSA Complete time to be larger than that of the current system. As additional beds are considered for the future system configuration, this performance measure is reduced. At a total of 18 beds in the future configuration, the performance measures become close to those of the current system.

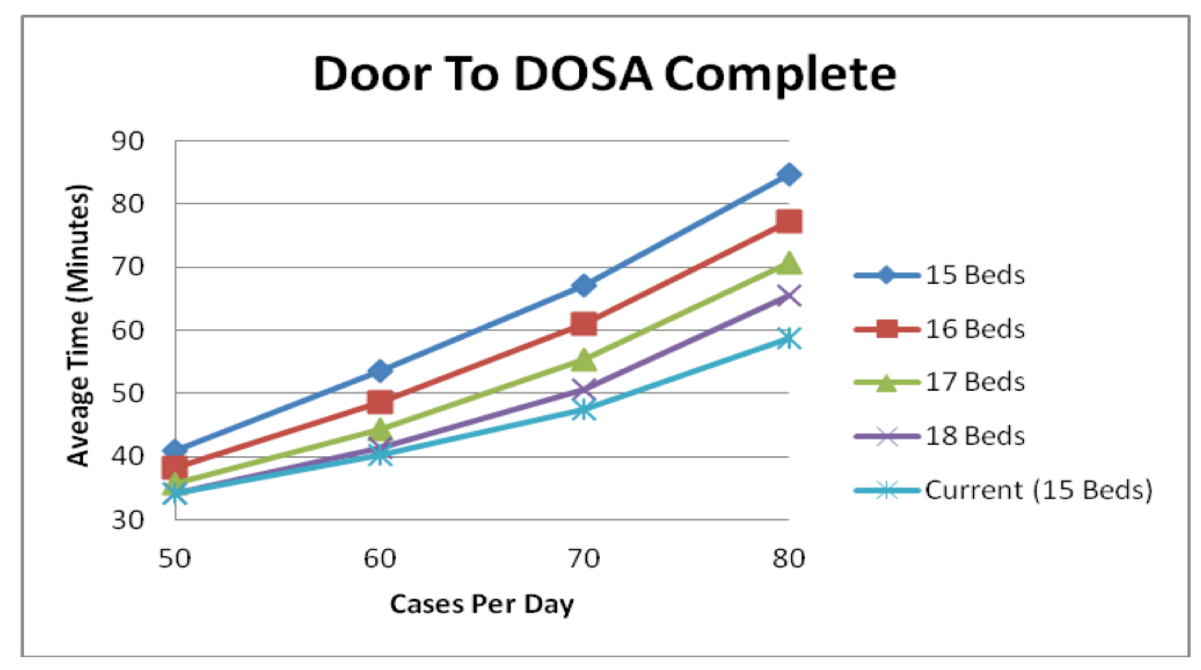

Figure 2: Comparison of Door to DOSA Complete time for the future system with 15 to 18 beds versus the current system for 50 to 80 scheduled surgery cases per day. 


\section{Kuhl}

Table 1 shows a comparison of the performance measures for the current system and those of the future system configuration containing 18 beds under various daily case loads. One can observe from the table that the overall performance of the future system with 18 beds is expected to perform very similar to that of the current system without the need for a separate holding area.

Table 1: Comparison of average performance measures of the current vs. the future DOSA configuration for fixed number of cases per day

\begin{tabular}{lrrrr}
\hline & \multicolumn{4}{c}{ Cases per Day } \\
\cline { 2 - 5 } Current System & \multicolumn{1}{c}{$\mathbf{5 0}$} & $\mathbf{6 0}$ & $\mathbf{7 0}$ & \multicolumn{1}{c}{$\mathbf{8 0}$} \\
\hline Door To DOSA Complete (min.) & 34.18 & 40.32 & 47.54 & 58.87 \\
Door To OR (min.) & 158.18 & 159.20 & 158.60 & 161.80 \\
Patient Wait In DOSA (min.) & 84.36 & 80.35 & 73.29 & 65.78 \\
Patient Wait In Holding (min.) & 16.99 & 16.09 & 15.34 & 14.48 \\
Holding Wait For Patient (min.) & 2.45 & 3.60 & 5.02 & 7.62 \\
OR Wait For Patient (min.) & 4.51 & 4.89 & 5.10 & 5.93 \\
Cases Remaining DOSA/Holding & 1.97 & 2.73 & 3.17 & 3.52 \\
Nurse Utilization & 0.27 & 0.32 & 0.36 & 0.40 \\
Tech Utilization & 0.46 & 0.51 & 0.55 & 0.59 \\
\hline & \multicolumn{4}{c}{ Cases per Day } \\
Future System (18 Beds) & $\mathbf{5 0}$ & $\mathbf{6 0}$ & $\mathbf{7 0}$ & $\mathbf{8 0}$ \\
\hline Door To DOSA Complete (min.) & 34.23 & 41.31 & 50.77 & 65.54 \\
Door To OR (min.) & 158.80 & 159.72 & 159.23 & 162.97 \\
Patient Wait In DOSA (min.) & 84.23 & 79.37 & 70.46 & 60.83 \\
Patient Wait In Holding (min.) & 22.73 & 21.65 & 20.42 & 18.79 \\
Holding Wait For Patient (min.) & 2.34 & 3.61 & 5.49 & 9.34 \\
OR Wait For Patient (min.) & 2.18 & 2.41 & 2.68 & 3.43 \\
Cases Remaining In DOSA & 1.39 & 1.79 & 2.21 & 2.32 \\
Nurse Utilization & 0.27 & 0.32 & 0.36 & 0.41 \\
Tech Utilization & 0.47 & 0.51 & 0.55 & 0.59 \\
\hline & \multicolumn{3}{c}{}
\end{tabular}

Finally, an additional experiment is conducted to compare system performance measures for the current and future DOSA unit configurations utilizing case loads that are randomly sampled from the distribution of case loads observed in the current system. The model of each system configuration was run for 100 replications utilizing the same random patient demands. The results are shown in Table 2. From this preliminary experiment, we can conclude that the future system with 18 beds where the holding function is performed within the DOSA unit will perform similar to current system.

Although this future system configuration is expected to perform similar to the current system under current case loads and performance levels of the other aspects of the perioperative process, the future plans may include the addition of additional operating rooms and an increased case load. Therefore, explicitly modeling the flow of the other perioperative processes will be necessary to accurately determine the optimal configuration for DOSA. In addition, in doing this, one may also be able to identify additional opportunities to improve productivity and efficiency. 
Table 2: Comparison performance measures of current and future DOSA unit configurations using daily case loads randomly sampled from the current observed distribution

\begin{tabular}{lrrrrrr}
\hline & \multicolumn{2}{c}{ Current System } & & \multicolumn{2}{c}{ Future System } \\
\cline { 2 - 3 } \cline { 6 - 7 } & Average & $\mathbf{9 5 \%}$ CI & & Average & $\mathbf{9 5 \%}$ CI \\
\hline Case Load & 43.62 & 1.88 & & 43.62 & 1.88 \\
Door To DOSA Complete (min.) & 33.12 & 1.02 & & 33.30 & 1.00 \\
Door To OR (min.) & 161.77 & 1.73 & & 162.33 & 1.75 \\
Nurse Utilization & 0.24 & 0.01 & & 0.24 & 0.01 \\
Tech Utilization & 0.39 & 0.01 & & 0.40 & 0.01 \\
\hline
\end{tabular}

\section{CONCLUSION}

In this paper, we presented a case study of a day of surgery admission unit for a relatively large hospital. From the preliminary experiment, we were able to conclude that the future system configuration where the OR Holding functions are preformed in the DOSA unit can be accomplished with 18 beds resulting in similar average performance as the current system. However, additional experimentation is needed to identify the critical factors that drive the performance of the DOSA unit as well as to evaluate the capabilities and limitations of the unit in terms of future patient volume.

\section{ACKNOWLEDGMENTS}

The author acknowledges the healthcare professionals at Rochester General Hospital for the opportunity to conduct this study and for their support in providing information, data, and direction in the development of the simulation models and the associated analysis.

\section{REFERENCES}

Ballard, S.M., and M.E. Kuhl. 2006. The Use of Simulation to Determine Maximum Capacity in the Surgical Suite Operating Room. In Proceedings of the 2006 Winter Simulation Conference, ed. L. F. Perrone, F. P. Wieland, J. Liu, B. G. Lawson, D. M. Nicol, and R. M. Fujimoto. Piscataway, New Jersey: Institute of Electrical and Electronics Engineers, 433-438.

Denton, B.T., A.J. Miller, H.J. Balasubramanian, and T.R. Huschka. 2010. Optimal Allocation of Surgery Blocks to Operating Rooms Under Uncertainty, Operations Research, 58(4), 802-816.

Marjamaa, R., P. Torkki, E. Hirvensalo, and O. Kirvelä. 2009. What is the Best Workflow for an Operating Room? A Simulation Study of Five Scenarios, Health Care Management Science, 12(2), 142-146.

Segev, D., R. Levi, P. Dunn, and W. Sandberg. 2012. Modeling the Impact of Changing Patient Transportation Systems on Peri-operative Process Performance in a Large Hospital: Insights from a Computer Simulation Study. Health Care Management Science, 15(2), 155-169.

\section{AUTHOR BIOGRAPHY}

MICHAEL E. KUHL is a Professor in the Industrial and Systems Engineering Department at Rochester Institute of Technology. He has a Ph.D. in Industrial Engineering from North Carolina State University (1997). His research interests include input modeling for simulation and simulation modeling methodologies with application to healthcare, manufacturing, logistics, and sustainability. He served as Proceedings Editor for the 2005 Winter Simulation Conference and will serve as Program Chair for the 2013 Winter Simulation Conference. He is a member of the INFORMS Simulation Society, IIE and ASEE. His e-mail address is <Michael.Kuhl@rit.edu> and his Web address is <people.rit.edu/mekeie>. 